\section{Training and testing effects in the generalization of a voluntary response}

\author{
JOHN A. HEBERT, DAVID P. ORIGLIO, and FRANK D. McGUIRK \\ Colorado State University, Fort Collins, Colo. 80521
}

The effects of amount of training on the generalization of a voluntary response were investigated under two testing procedures-one in which the original training stimulus, a $100-\mathrm{g}$ weight, was presented five times more frequently than the other test stimuli, and another in which the original training stimuluswas presented with the same frequency as the other test stimuli. No effects of amount of training were observed, in support of previous findings. However, the two procedures produced different-shaped gradients of stimulus generalization, also in support of previous findings.

A study by White (1965) on the generalization of a voluntary response showed that varying the amount of training on the original stimulus $\left(S_{1}\right)$ did not have any differential effect on the subsequent generalization (GSGs), either in the form of differing slopes or of shape. Using wavelength as a stimulus dimension, White presented a red stimulus $\left(S_{1}\right)$ for a brief time, and then varied the amount of training of $S_{1}$ from 0 to 30 additional trials. Testing in the direction of shorter wavelengths, White found that all training groups responded maximally at $S_{1}$ and that they showed a decrease in response as similarity of the test stimulus to $S$, decreased, rather typical GSGs. Thus, amount of training did not appear to be an important variable in determining the slope or shape of the GSG. However, White used a procedure in testing which may have directly affected the obtained GSGs: $S_{1}$ was presented approximately five times more frequently in testing than were the other four test stimuli. Hebert \& Capehart (1969), using a weight dimension, have shown that this procedure of "overrepresenting" $S$, in testing yields a GSG of the type White (1965) reported, but that a procedure in which $S_{1}$ is presented with the same frequency as the other test stimuli produces a much different GSG: Maximal responding to a stimulus adjacent to $S_{1}$, not to $S_{1}$ itself, yielded a somewhat curvilinear gradient. This "central-tendency effect" has been described previously with a wavelength dimension (Thomas \& Jones, 1962) and with a size-area dimension (Helson \& Avant, 1967). The studies cited above which reported a central-tendency effect did not vary amount of training as did White (1965) and, in fact, used very minimal amounts of training ( 1 to 5 trials).

The present study was designed to investigate two questions: (1) Does the procedure of overrepresenting $S_{1}$ in testing mask the effects of amount of training on the GSG (effects which might otherwise be observed with an equal-presentation procedure)? (2) Would the central-tendency effect be observed with larger amounts of training than in previous studies?

\section{METHOD}

Ninety students from an introductory psychology class served as Ss. Experimental treatments were assigned to $\mathrm{Ss}$ on a random basis as they showed up for the experiment. The five stimuli, ranging in weight from 100 to $200 \mathrm{~g}$ in $25 \mathrm{~g}$ steps, were made from aluminum cans, 4 in. tall and 2 in. in diam, which were filled with wax and shot and painted to appear identical. When a single weight was lifted with S's right hand, a microswitch which started a timer was released. When $\mathrm{S}$ reached his decision, he pressed one of two response keys with the left index finger which rested above and between the two closely spaced response keys. If $S$ felt that a test stimulus was the same as $S_{2}$, he was instructed to press the "same" key and, if otherwise, to press the "different" key. Both choice and decision times were recorded by $E$. The blindfolded Ss were trained on the 100 -g stimulus for 1,11 , or 21 trials. A trial consisted of S's grasping the weighted can from the top with four fingers and thumb, lifting the weight straight up (with elbow resting on the table), and bringing it straight down and releasing it. Shaking or swinging the weight was not permitted. At the completion of training, each of the three training groups of $30 \mathrm{Ss}$ each was further subdivided into two subgroups of $15 \mathrm{Ss}$ each. One of the subgroups within each training group received the overrepresentation procedure in testing, while the other subgroup received the equal-presentation procedure. The overrepresentation subgroups ( $O$ groups) were given a test series of four blocks of 9 trials each (five presentations of $S_{1}$ and one each of the other four test stimuli per equal-presentation subgroups (E block), or 36 trials. The groups) were given four blocks of 5 trials each (one presentation of each of the five test stimuli per block), or 20 trials. Within each block, stimuli were presented in a random order with the restriction that $S$ be presented no more than twice in a row. The design of the study was a 3 by 2 by 5 factorial analysis of variance with repeated measures, with three levels of training, two testing procedures, and five stimuli as the repeated measures variable. The proportion of times a given stimulus was chosen as the "same" was calculated and transformed by an arcsin method ( $\mathrm{X}^{\prime}=$ $2 \arcsin \sqrt{X}$ ) to serve as the dependent measure for choice behavior.

RESULTS AND DISCUSSION

The finding of White (1965) that amount of training does not affect the slope or shape of the GSG is also observed in this study. The three training groups $(1,11$, and 21 trials) yielded similar GSGs under both procedure conditions: a downward linear GSG in the overrepresentation groups with peak response at $S_{1}$, and in the equal-presentation groups, a somewhat curvilinear GSG with an

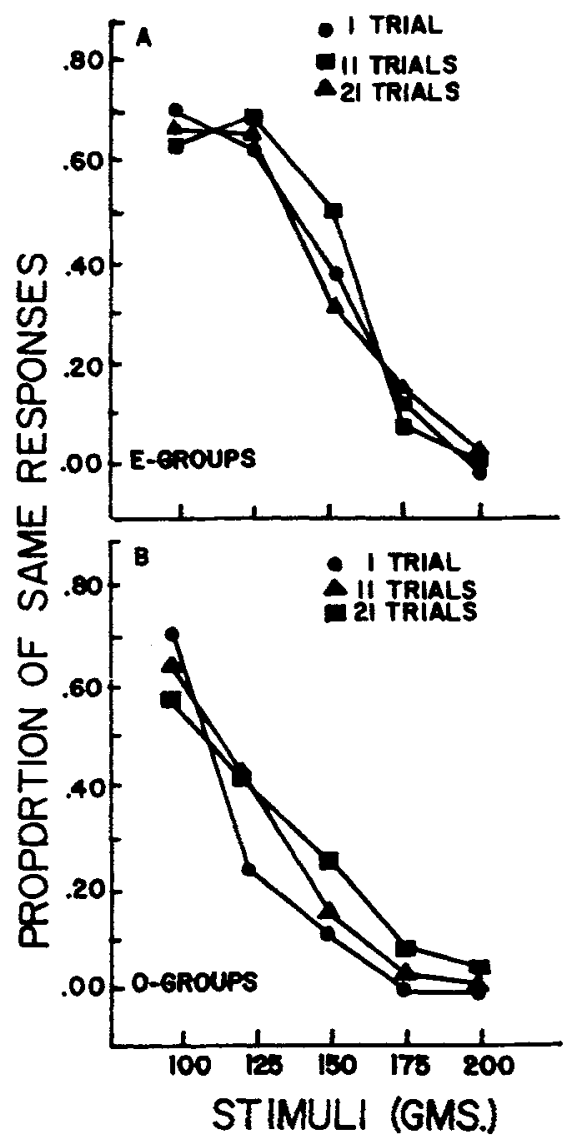

Fig. 1. Gradients of stimulus generalization for the equal presentation groups (A) and the overrepresentation groups (B). 

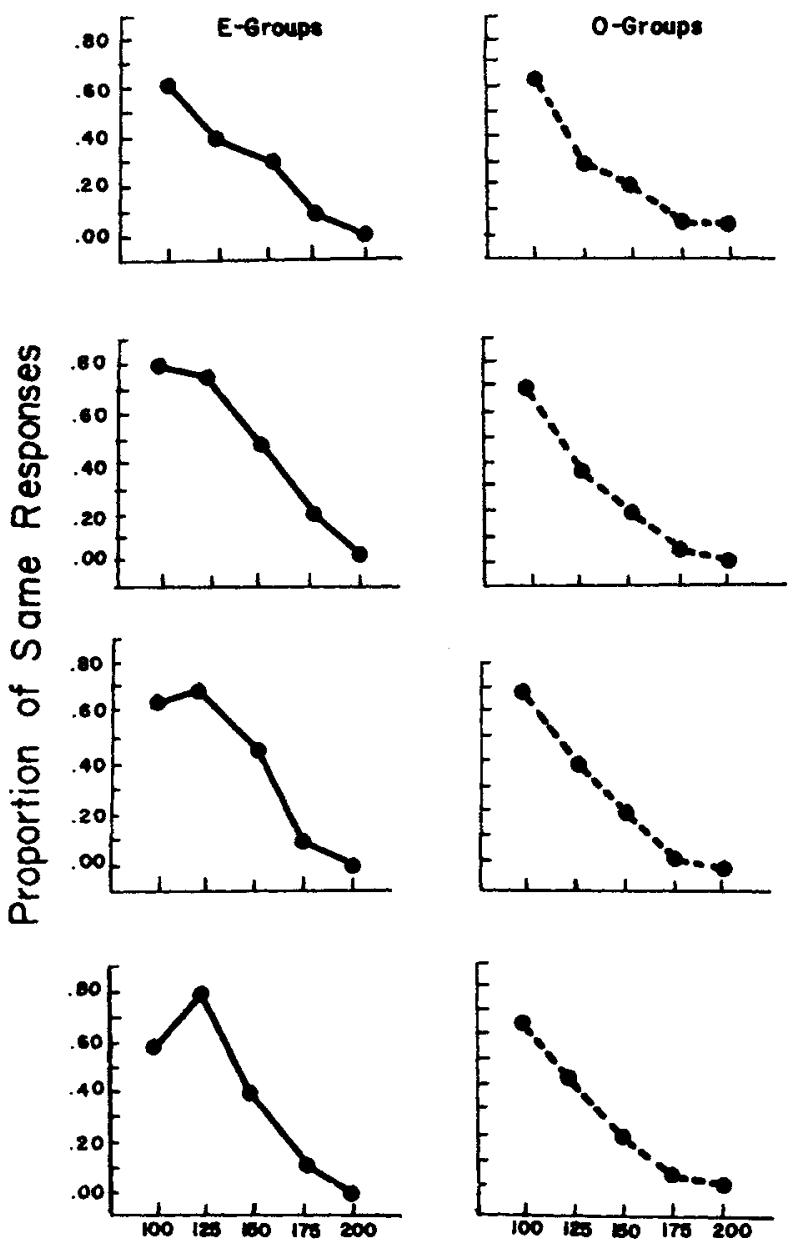

\section{Stimuli (Grams)}

Fig. 2. Gradients of stimulus generalization shown in successive fourths of testing (top to bottom), separately for equal presentation groups (solid line) and overrepresentation groups (dashed line).

increased tendency to choose $S_{2}$ and $\mathrm{S}_{3}$ as the original. Figure 1 shows the GSGs for all six experimental groups. The absence of significant Stimuli by Training $(F=1.87, \quad d f=8 / 336$, $\mathrm{p}>.05)$ and Stimuli by Training by Procedure $(F=.62, \mathrm{df}=8 / 336$, $p>.05)$ interactions indicate the absence of training effects. The nonsignificant Stimulus by Procedure by Training interaction can be interpreted to mean that increased amounts of training did not retard the shift from $S_{1}$ to $S_{2}$. This is somewhat inconsistent with the adaptation-level (AL) interpretation given to similar data by Capehart, Tempone, \& Hebert
(1969). Presumably, increased frequency of $S_{1}$ in training should retard the shift in the same fashion as increased frequency of $S_{1}$ in testing. One possible explanation for this not occurring is that $S_{1}$ is actively judged in testing but, in training, no judgment about the weight is required of $S$ on the additional training trials. A training procedure in which $\mathbf{S}$ actively judges $S_{1}$ (e.g., rating of weight, discrimination training) might have a more pronounced effect on test data. Finally, the absence of a significant training main effect $(F=1.89$, $\mathrm{df}=2 / 84, \quad \mathrm{p}>.05)$ indicates that overall level of response is not affected by amount of training. Thequestion of procedure masking any training effects, then, is eliminated since there are no discernible training effects under either procedure.

The second question of obtaining the central-tendency effect with larger amounts of training than in previous studies is answered affirmatively. All three equal-presentation groups showed an increased tendency to prefer intermediate value stimuli when compared with the overrepresentation groups (Fig. 1). Figure 2 shows that this central tendency in the equal-presentation groups develops gradually as a function of test experience. The significant Stimuli by Procedure interaction $(F=6.15$, $\mathrm{df}=4 / 336, \mathrm{p}<.001$ ) is depicted in successive blocks of test trials. At the onset of testing (top of Fig. 2) the equal-presentation groups ( $E$ groups) and the overrepresentation groups (O groups) respond in a similar fashion, but as testing proceeds (top to bottom in Fig. 2) the $\mathbf{E}$ groups show a shift in the GSG from a linear to a curvilinear trend, whereas the $O$ groups maintain the same linear trend throughout testing. Trend analysis of this Stimulus by Procedure interaction shows that the $O$ and $E$ groups do not differ in their linear trends $(F=2.14$, $\mathrm{df}=1 / 336, p>.05$ ) but differ significantly in their curvilinear trends $(\mathrm{F}=18.40, \quad \mathrm{df}=1 / 336, \quad \mathrm{p}<.001)$, confirming that the difference in the GSGs of the two groups relates to differences in shape of the gradient.

In summary, this study has replicated the findings of the Hebert \& Capehart (1969) study, and has extended those findings to conditions involving differential amounts of training.

\section{REFERENCES}

CAPEHART, J. E., TEMPONE, V., \& HEBERT, J. A. A theory of stimulus equivalence. Psychological Review, 1969, $76,405-413$.

HEBET J A \& CAPEHART, J. E. Generalization of a voluntary response as a function of presentation frequency of the training stimulus in testing. Psychonomic Science, 1969, 16, 315-316. HELSON, H. \& AVANT, L. L. Stimulus generalization as a function of contextual stimuli. Journal of Experimental Psychology, 1967, 73, 565-567.

THOMAS, D. R., \& JONES, C. G. Stimulus generalization as a function of the frame of reference. Journal of Experimental Psychology, 1962, 64, 77-80.

WHITE, $S$. Training and timing in the generalization of a voluntary response. Journal of Experimental Psychology, $1965,69,269-275$. 